\title{
Passive acoustic measurement of flow velocity in the Straits of Florida
}

\author{
Oleg A Godin ${ }^{1 *}$, Michael G Brown², Nikolay A Zabotin ${ }^{3}$, Liudmila Y Zabotina ${ }^{3}$ and Neil J Williams²
}

\begin{abstract}
Group speed of sound in moving fluids depends on the propagation direction, which breaks acoustic reciprocity. Acoustic nonreciprocity provides a means to measure fluid motion. Using nonreciprocity, one can measure fluid velocities that may be small compared to uncertainties in sound speed. Interferometry of diffuse acoustic noise, with receivers replacing the transceivers employed in active techniques, offers a simple, low-cost means of measuring nonreciprocity. Here, the feasibility of using passive measurements of acoustic nonreciprocity to estimate current velocity in the ocean is experimentally demonstrated for the first time. Estimates of depth-averaged flow velocity are retrieved from cross-correlations of low-frequency noise recorded in the Straits of Florida by near-bottom hydrophones separated by 5 and $10 \mathrm{~km}$.
\end{abstract}

Keywords: Noise interferometry; Ocean acoustic tomography; Acoustic oceanography; Reciprocity in acoustics; Florida current; Passive remote sensing

\section{Background}

Measurements of velocity of oceanic currents are crucial for evaluating heat and mass transport, monitoring ocean dynamics, and understanding the ocean's role in climate. Reciprocal transmissions of acoustic signals, i.e., sound propagation in opposite directions between two points, provide a way to measure path-averaged current velocity in the ocean with high temporal resolution, on a large scale, and over extended periods of time [1-10]. This approach is based on measurements of acoustic nonreciprocity, which is defined as differences between travel times or other acoustic quantities corresponding to sound propagation in opposite directions between two points. Acoustic nonreciprocity, which vanishes in motionless media, is a direct, sensitive measure of fluid motion, and is insensitive to uncertainties in the sound speed field and geometry of the experiment $[4,5,11]$.

The use of active reciprocal transmissions for ocean remote sensing applications is limited by capital and operational costs associated with low-frequency acoustic sources as well as concerns about potential impacts of transmissions on marine life. Wave interferometry [12]

\footnotetext{
* Correspondence: oleg.godin@noaa.gov

${ }^{1}$ Cooperative Institute for Research in Environmental Sciences, University of Colorado and NOAA Earth System Research Laboratory, Physical Sciences

Division, Boulder, Colorado 80305-3328, USA

Full list of author information is available at the end of the article
}

and, in particular, underwater acoustic noise interferometry [13-17] offers a means to perform passive remote sensing by replacing probing signals generated by a dedicated source with two-point cross-correlations of diffuse ambient noise. Theory $[18,19]$ predicts that the cross-correlation function of diffuse noise measured at two locations in a generic inhomogeneous moving medium results in approximations to Green's functions describing sound propagation in opposite directions between the two measurement points; positive and negative lags correspond to propagation in opposite directions [18-20]. Thus, two-point correlation functions allow one to quantify flow-induced acoustic nonreciprocity. Recently, passive measurements of acoustic nonreciprocity and wind velocity were experimentally demonstrated in atmospheric acoustics using interferometry of traffic noise [20].

In this paper, we use the data obtained in a noise interferometry experiment [17] in the Straits of Florida to investigate the feasibility of passive remote sensing of currents in the ocean. We present the first experimental demonstration that acoustic nonreciprocity induced by oceanic currents can be measured by noise interferometry and that the current velocity can be retrieved from the acoustic noise cross-correlations. 


\section{Methods}

Ambient and shipping noise in the Florida Straits was recorded by three autonomous systems situated on the continental shelf approximately along the $100 \mathrm{~m}$ isobath $15 \mathrm{~km}$ off the Florida Keys (Figure 1). Seafloor slope across the isobaths was of the order of $10^{-2}$. Horizontal separations of the instruments were $5.01,9.76$, and $14.76 \mathrm{~km}$ for the $1-2,2-3$, and $1-3$ instrument pairs, respectively. The three instruments approximately fell on a straight line with 1 northernmost and 3 southernmost. Each system had a hydrophone, electronics, and batteries contained in an aluminum housing on a short, taut mooring, with the hydrophone located $5 \mathrm{~m}$ off the seafloor. The electronics and recording components were based on units commercially available from Loggerhead Instruments, but the default system clock was replaced with a more accurate crystal oscillator. The nominal clock drift was $10^{-8}$, or about $1 \mathrm{~ms} /$ day.

The instruments were deployed on December 12-13, 2012 and retrieved three months later. Because of an electronics malfunction in one of the instruments on the seventh post-deployment day, only about six days of continuous, simultaneous records by all three systems are available. Those six days of data are used in this paper to calculate cross-correlations of acoustic noise between various hydrophone pairs. CTD (conductivity, temperature, depth) casts were performed on the deployment and recovery cruises. Temperature variations with depth and sound speed gradients were rather weak in December 2012, with the sound speed $c=1537.4 \pm 2.4 \mathrm{~m} / \mathrm{s}$ throughout the water column. During the experiment, tides with a total range of about $1 \mathrm{~m}$ were recorded on a tide gauge at Key Biscayne, 68-81 km from the instruments.

Ship-based ADCP (acoustic Doppler current profiler) measurements were made on the deployment cruise using a dual frequency $(600 \mathrm{kHz}$ and $75 \mathrm{kHz}$ ) system; see Figure 1. As shown in the figure, the depths covered by these instruments are approximately $2-10 \mathrm{~m}$ and $10-70 \mathrm{~m}$, respectively. The $70 \mathrm{~m}$ lower limit is imposed by the sidelobe structure of the transmitted $75 \mathrm{kHz}$ beam coupled with reflections off the bottom at $100 \mathrm{~m}$. The time intervals during which the ship was in proximity to our instruments are marked on the figure. At those times/ locations the depth-averaged eastward and northward current components are approximately $0.4 \mathrm{~m} / \mathrm{s}$ and $0.6 \mathrm{~m} /$ $\mathrm{s}$, respectively, corresponding to an average current speed of $0.7 \mathrm{~m} / \mathrm{s}$, directed approximately 55 degrees north of east. This direction roughly coincides with the orientation (instrument 3 to instrument 1) of our array. Currents in the lower $30 \mathrm{~m}$ of the water column are presumably lower, but we have no quantitative estimate of those currents, and we have also have no information on the postdeployment temporal evolution of currents at any depth.

Acoustic pressure was recorded with $8 \mathrm{kHz}$ sampling frequency. However, only the low-frequency part of the noise spectrum proved to be useful for noise interferometry. At ranges of 5 to $15 \mathrm{~km}$, averaging times longer than the M2 tidal period are needed for deterministic features to emerge in the two-point correlation functions of noise. Tidally induced ocean-depth variations lead to a loss of coherence between the noise sampled by the hydrophones [17]. Coherence loss due to tidal fluctuations rapidly
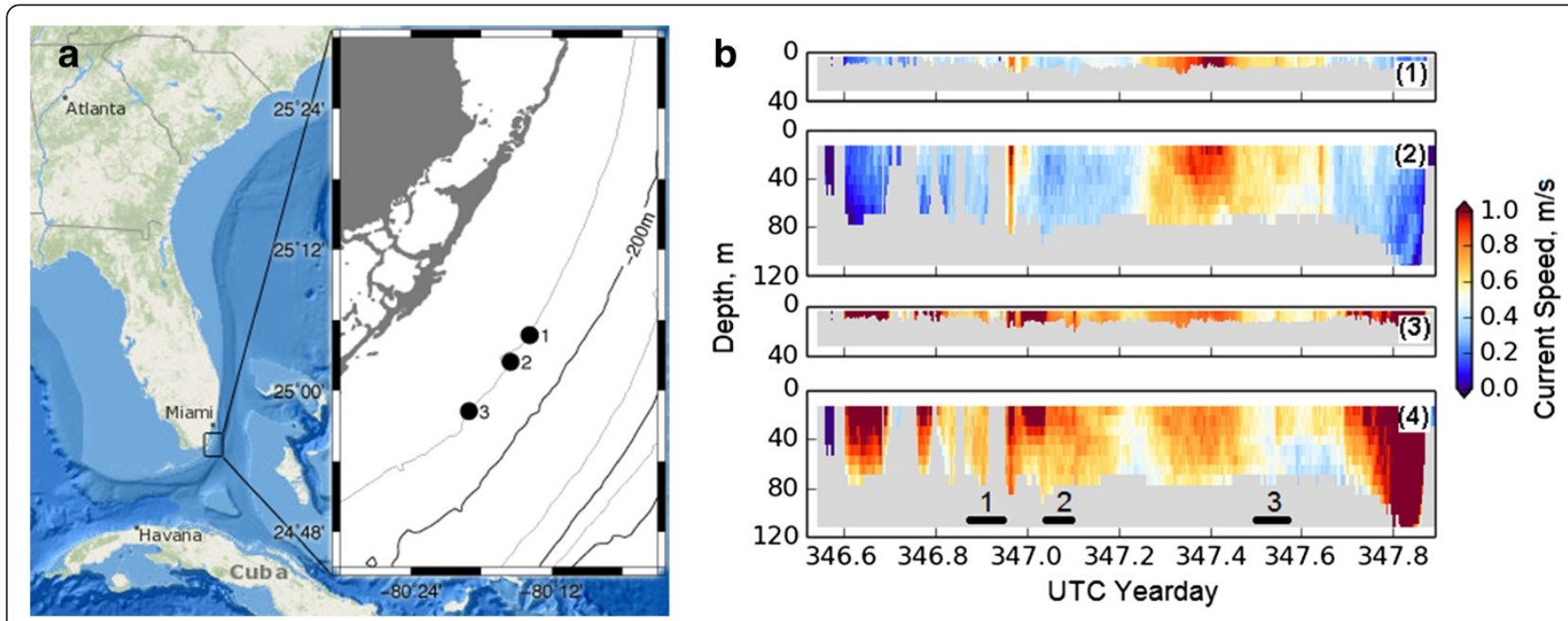

Figure 1 Acoustic noise interferometry experiment in the Florida Straits. (a) Maps show the observation area with bathymetry contours and positions (black circles) of the autonomous noise recording systems 1, 2, and 3 deployed in December 2012 . The contour interval is 100 m. (b) Shipborne dual-frequency acoustic Doppler current profiler (ADCP) measurements, from the deployment cruise, of the eastward (panels 1 and 2) and northward (panels 3 and 4) components of current velocity. 600 kHz measurements (panels 1 and 3) yield current estimates in the 2-10 m depth range, while $75 \mathrm{kHz}$ measurements (panels 2 and 4) yield current estimates in the 10-70 m depth range. Three time intervals, when the ship was in the vicinity of the noise recording systems, are indicated by black bars. 
increases with acoustic frequency and the number of surface reflections. In agreement with our observations, theoretical estimates predict almost total loss of coherence above about $80 \mathrm{~Hz}$ at $r=5 \mathrm{~km}$ and at even lower frequencies at longer ranges [17]. In this paper, we focus on the 20-50 Hz band, where coherence loss caused by tidal fluctuations is moderate and does not prevent emergence of deterministic features from noise cross-correlations (Figure 2). In this band the frequency response of the hydrophones is flat, and the acoustic field is less sensitive to seafloor structure than the field at lower frequencies.

Clock stability is a key requirement in both active and passive ocean remote sensing as a sub-millisecond accuracy is necessary for measurements of $O(1) \mathrm{m} / \mathrm{s}$ flow velocities and sound speed variations. In our experiment, the three instrument clocks were synchronized prior to deployment by simultaneously transmitting a pulse to all three instruments. (A post-recovery synchronization pulse was also employed, but, because of the aforementioned electronics malfunction, could not be used.) In a different deployment of the same instruments in the Florida Straits in September-October 2013 relative clock drifts obtained from the pre-deployment and post-recovery clock synchronization were $1.189 \mathrm{~ms} /$ day for the 1-2 instrument pair and $-0.774 \mathrm{~ms} /$ day for the $2-3$ instrument pair. Acoustic data obtained in the September-October 2013 experiment will not be discussed in this paper.

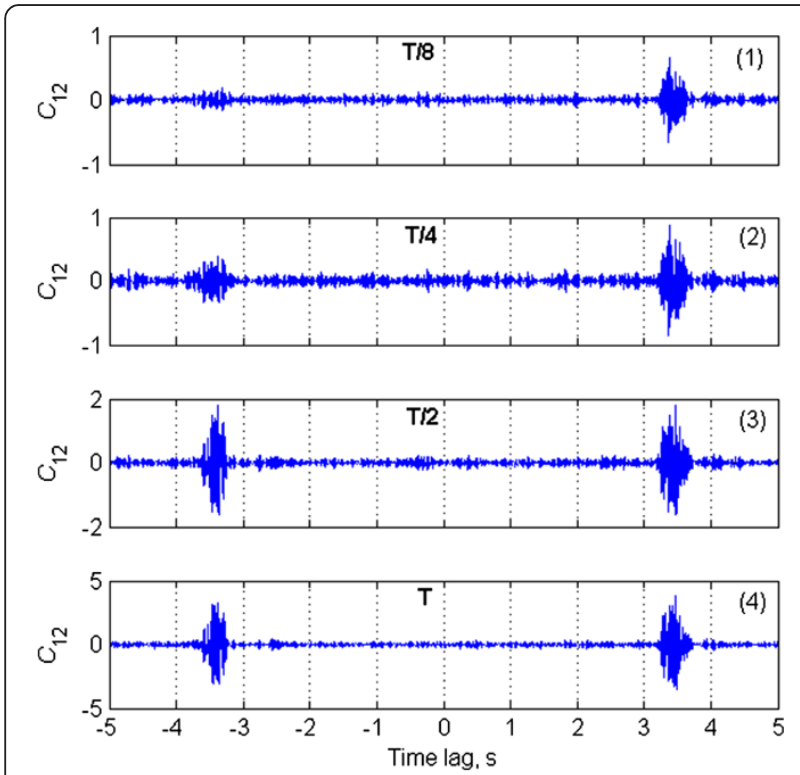

Figure 2 Emergence of a coherent structure from underwater acoustic noise. Estimates of the noise cross-correlation function $C_{12}$ for the 1-2 path are obtained using various averaging times. The noise averaging time doubles for each subsequent panel and varies from $1 / 8$ of the available data record to the entire record $T \approx 6$ days. The estimates of the noise cross-correlation function are shown in relative units. Note the difference between the scales used for $C_{12}$ in different panels.
A dedicated laboratory experiment was conducted in May 2014 to investigate the stability of the clocks in our instruments. Over a four-week period, seventeen clock synchronization pulses were transmitted simultaneously to the three instruments. The relative drifts were $1.521 \mathrm{~ms} /$ day for the 1-2 instrument pair and $-1.452 \mathrm{~ms} /$ day for the 2-3 instrument pair (Figure 3). In this test, deviations from a linear growth of clock time differences, i.e., deviations from constant clock drift, were small; these residual errors were less than $0.10 \mathrm{~ms}$ over all 1 week intervals for all clock pairs.

To evaluate cross-correlations of underwater noise, measurements of pressure fluctuations on each instrument were split into $625 \mathrm{~s}$ segments. Statistical averaging was approximated by averaging over a large number of data segments. For each hydrophone, $4.55 \%$ of data segments with the highest average noise level were discarded to suppress contributions of strong, localized, transient sources. (For a random variable with a Gaussian distribution, this corresponds to discarding the outliers, which exceed the mean by more than two standard deviations). Power spectra of the recorded noise have multiple sharp peaks, which is typical of shipping noise. To suppress the effects of a rapid variation of the noise power spectrum with frequency, we followed Godin et al. [21] and evaluated the noise cross-correlation as a Fourier transform of the coherence function, rather than the cross-spectrum. This is equivalent to pre-whitening and normalization of the noise spectrum in each data segment and can be viewed as a frequency-domain counterpart of "one-bit correlation" in the time domain [12], which has been extensively utilized in noise interferometry.

\section{Results and discussion}

Figure 2 illustrates the gradual emergence of deterministic features from cross-correlations of acoustic noise with increasing averaging time. For the 1-2 instrument pair, coherent features at positive and negative time lags emerge after less than 18 hours of noise averaging. Generally, the signal-to-noise ratio (SNR), i.e., the amplitude contrast between the coherent features (with time lags between about $3.2 \mathrm{~s}$ and $3.7 \mathrm{~s}$ ) and the spurious arrivals, increases with increasing averaging time. Small differences in amplitude and time shifts of the fine structure of the correlation functions at positive and negative lag are seen. The amplitude differences can be attributed to noise anisotropy. Comparison of $C_{12}$ with the cross-correlation functions $C_{23}$ and $C_{13}$ for the 2-3 and 1-3 instrument pairs shows that, with increasing instrument separation, SNR decreases and longer averaging times become necessary.

In noise interferometry, the positive [negative] time lag parts of a noise cross-correlation function $C_{i j}$ are approximations to the Green's functions describing propagation from location $i$ to location $j$ [location $j$ to location $i$ ]. In 

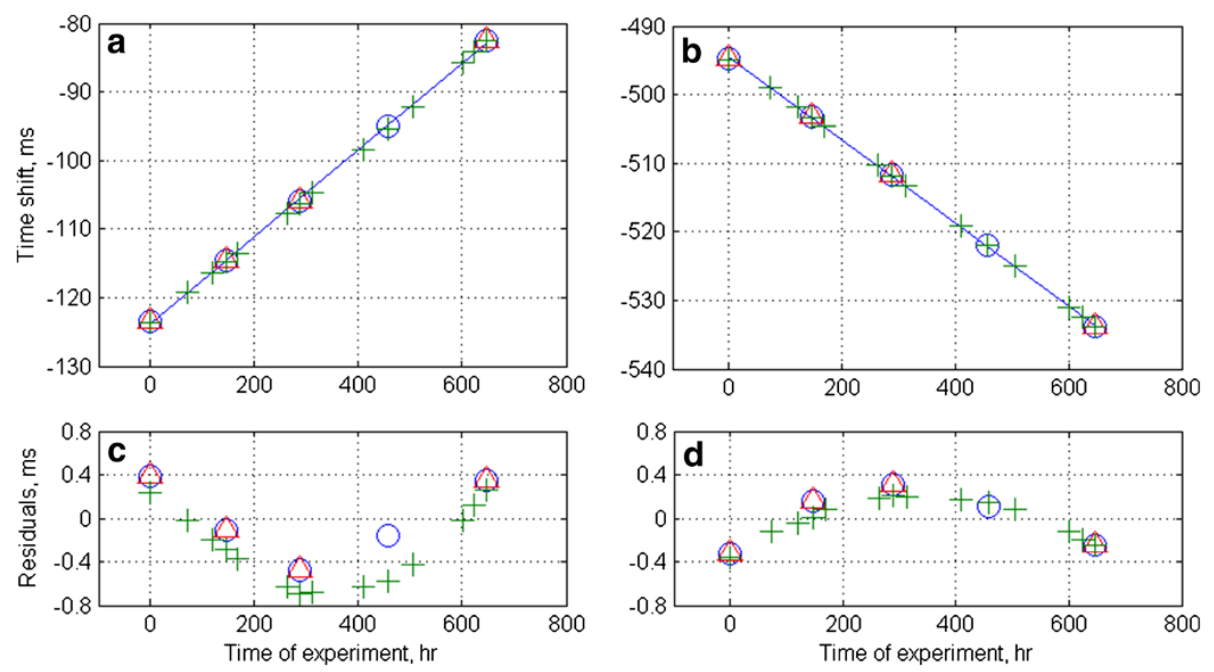

Figure 3 Laboratory experiment on clock stability. (a) Relative time shift between the system clocks of instruments 1 and 2 is shown as a function of time from the start of the experiment. The linear fit to the measurements corresponds to the assumption that the relative clock drift is constant over 4 weeks. (b) Same as (a) but for instruments 2 and 3. (c) Deviation of measured time shifts from the average linear month-long trend for the 1-2 clock pair. (d) Same as (c) but for instruments 2 and 3. Circles, triangles, and crosses show the measurements using noise cross-correlation, cross-correlation of synchronization pulses, and matching the leading fronts of the synchronization pulses, respectively.

our data $C_{i j}(t)$ and $C_{i j}(-t)$ are nearly identical (owing to an approximately diffuse distribution of noise sources), apart from a time shift. In the following we estimate the time shift between $C_{i j}(t)$ and $C_{i j}(-t)$ by identifying the location of the peak in the cross-correlation function between $C_{i j}(t)$ and $C_{i j}(-t)$. (It is important not to confuse this secondary correlation function calculation with the process by which the $C_{i j}$ were estimated - cross-correlating records of ambient noise measured at locations $i$ and $j$.) The time shifts between $C_{i j}(t)$ and $C_{i j}(-t)$ that we seek to estimate are due to a combination of acoustic travel time nonreciprocity and clock errors. To separate these effects, we exploit the properties that, over time scales that are not too long, clock errors grow linearly, while the nonreciprocity contribution is constant. The 6 day data set was divided into four consecutive segments of equal 1.5 day duration. For each segment, we evaluated the noise crosscorrelation functions $C_{i j}$ and calculated the normalized correlation function $K_{i j}$ of the measured noise crosscorrelations $C_{i j}(t)$ at the positive and negative time lags $t$ :

$$
K_{i j}(\tau)=\left[\int_{t_{1}}^{t_{2}} C_{i j}^{2}(t) d t \cdot \int_{t_{1}}^{t_{2}} C_{i j}^{2}(-t) d t\right]^{-1 / 2} \int_{t_{1}}^{t_{2}} C_{i j}(t) C_{i j}(\tau-t) d t .
$$

Results for the 1-2 and 2-3 instrument pairs are shown in Figure 4. (Low SNR prevents application of this analysis to $\left.C_{13}\right)$. The integration interval $\left(t_{1}, t_{2}\right)$ in Eq. (1) was chosen to contain the coherent features in the respective $C_{i j}(t)$. Peak values of $K_{i j}$ characterize the degree of similarity between $C_{i j}$ at positive and negative time delays. For perfectly diffuse noise, infinite averaging time, and weak currents the peak value equals unity. In addition to main peaks, each $K_{i j}(\tau)$ has additional peaks due to finite noise bandwidth. The position of each main peak gives an estimate of the time shift of the corresponding data segment.

The best-fitting lines to our two sets of four time shift estimates are shown in Figure 4c. The slopes of those lines, caused by clocks drifting at a near-constant rate, are $0.917 \mathrm{~ms} /$ day and $-0.490 \mathrm{~ms} /$ day for the $1-2$ and 2-3 instrument pairs, respectively. The zero-time (clock synchronization time) intercepts of those lines, corresponding to current-induced time shifts, are $\tau_{12}=-2.00 \mathrm{~ms}$ and $\tau_{23}=-4.10 \mathrm{~ms}$. We emphasize here that, although one cannot distinguish between current-induced and clockdrift-induced contributions to a time shift using an isolated estimate, the only sensible interpretation of the linear trends shown in Figure 4c is that over the 6 day measurement period the clocks were drifting at a nearconstant rate and the current was very nearly steady. (It would take a remarkable coincidence for two nonlinear trends to effectively cancel each other.) Additional support for the assumptions underlying our interpretation of Figure 4c is described below.

Special cases of the procedure that we have described to retrieve clock drift and travel time nonreciprocity from ambient noise cross-correlations have been previously employed in seismology [22-24], where there was no nonreciprocity, and in atmospheric acoustics [20], where there was no clock drift. To test our method of estimating time 

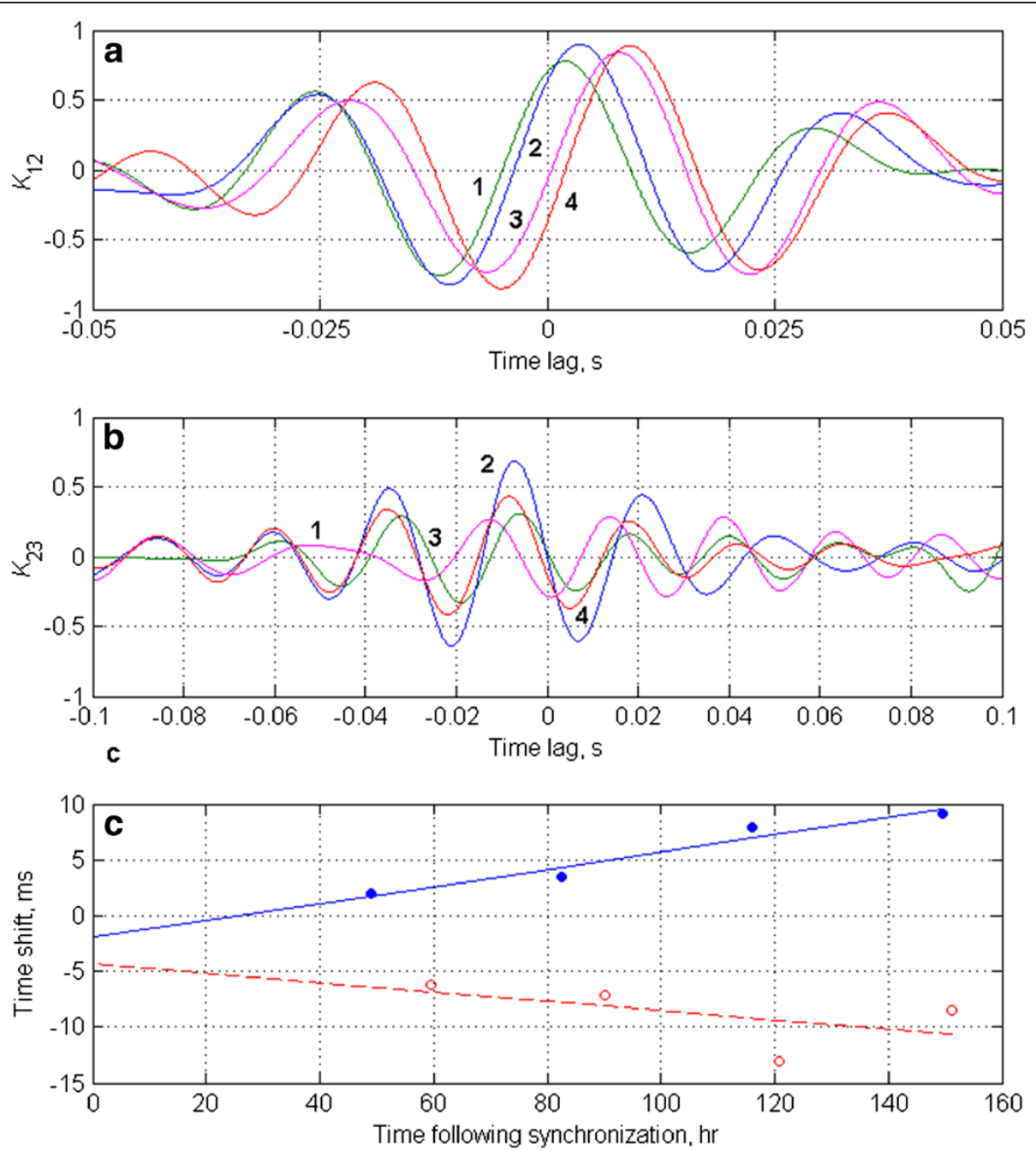

Figure 4 Measurements of the system clocks' drift using correlation of noise correlations. (a) Correlation between the positive- and negative-time-delay parts of the measured cross-correlation function $C_{12}$. The function $C_{12}(t)$ is correlated with $C_{12}(-t)$ for the time delays $3.2 \mathrm{~s}<$ $t<3.6 \mathrm{~s}$, which correspond to the deterministic acoustic arrivals. Line numbers indicate the quarter (first to fourth) of the available data set used in the $C_{12}$ calculation. (b) Same as in (a) but for the measured cross-correlation function $C_{23}$ for the $2-3$ pair and $6.2 \mathrm{~s}<t<7.0 \mathrm{~s}$. (c) Least-square fits to the time shifts between 1-2 (solid line) and 2-3 (dashed line) instruments measured at different geotimes. The geotime is shown in hours following the pre-deployment clock synchronization.

shifts, we applied it to the ambient noise recorded between synchronization pulses in the laboratory experiment on clock stability. The relative drifts of clocks measured by matching the leading fronts of the synchronization pulses and from cross-correlations of ambient noise were found to agree within $0.1 \%$ (Figures 3a, b). Our relative clock drift estimates based on Figure $4 \mathrm{c}$ are of the same magnitude as the clock drifts measured (i) in SeptemberOctober 2013 using pre-deployment and post-recovery clock synchronization pulses, and (ii) in the May 2014 laboratory experiment. The reported values of the relative clock drifts, including our estimates based on Figure 4c, are consistent with the clock drifts being nearly constant over time periods of the order of a few weeks and slowly varying over periods of the order of a few months.
Correlation functions $C_{12}$ and $C_{23}$, computed after having corrected for estimated clock drift and averaging over the entire 6-day data set, are shown in Figures $5 a$ and $b$. In those correlation functions clock-drift-induced blurring is eliminated, SNR is higher than in the 1.5 day averages, and the nonreciprocity time shift is isolated. The corresponding functions $K_{12}(\tau)$ and $K_{23}(\tau)$ shown in Figure 5c have peak values of 0.954 and 0.763 , respectively; the corresponding time shifts, $\tau_{12}=-2.00 \mathrm{~ms}$ and $\tau_{23}=-4.10 \mathrm{~ms}$, are in very good agreement (coincidentally identical here) with the estimates of the same quantities based on Figure 4c. As reflected by the peak values of $K_{12}$ and $K_{23}$, the uncertainty of the $\tau_{23}$ estimate is greater than that of the $\tau_{12}$ estimate. This difference is attributable to greater distance between the 2-3 instrument pair, and the 


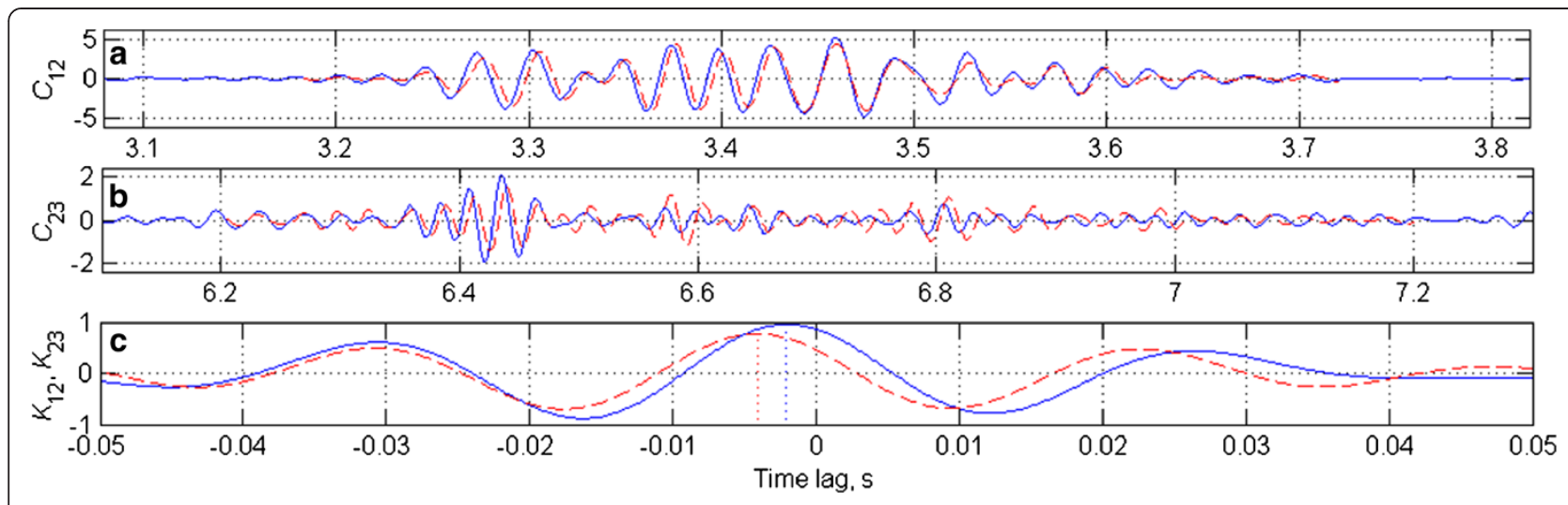

Figure 5 Comparison of the fine structure of noise cross-correlations at positive and negative time delays. (a) The noise cross-correlation function $C_{12}$ is shown for positive (solid line) and negative (dashed line) time delays after removal of the relative drift of system clocks. The entire available data set is used for noise averaging. (b) Same as (a) but for $C_{23}$. (c) Correlation between the positive- and negative-time-delay parts of the cross-correlation functions $C_{12}$ (solid line) and $C_{23}$ (dashed line). The position of the peak of the correlation of correlations determines the nonreciprocity of travel times induced by currents at sound propagation between the respective pair of instruments.

correspondingly lower SNR of the $C_{23}$ correlation function. In spite of the greater uncertainty of the $\tau_{23}$ estimate, note that the ratio of the distances between the $2-3$ and $1-2$ instrument pairs is $9.76 \mathrm{~km} / 5.01 \mathrm{~km}=1.95$, which is in good agreement with the ratio $\mathrm{T}_{23} / \mathrm{\tau}_{12}=2.05$ and the assumption of a constant current along the $100 \mathrm{~m}$ isobath.

Under conditions of the experiment, the acoustic Green's function for propagation between the instruments is composed of multiple surface- and bottom-reflected ray arrivals, and, in the $20-50 \mathrm{~Hz}$ frequency band that we consider, neither ray nor mode arrivals can be resolved in the time domain [17]. On an individual eigenray connecting points $\left(0,0, z_{A}\right)$ and $\left(r, 0, z_{B}\right)$, the difference between acoustic travel times from $B$ to $A$ and $A$ to $B$ is [4] $\delta t=2 \int_{0}^{r} u_{x} c^{-2} d x$ up to the terms of the third order in the ratio of the flow velocity $\mathbf{u}=\left(u_{x}, u_{y}, 0\right)$ and the sound speed $c$. The integration is along the eigenray traced in the absence of currents. By considering the straight segments of the eigenray between reflections, in a range-independent waveguide with a constant sound speed and depthdependent flow velocity $\mathbf{u}(z)$, the travel time nonreciprocity equation simplifies to $\delta t=2 c^{-2} r U, U=H^{-1} \int_{0}^{H} u_{x}(z) d z$, when both points are located on the seafloor. Here $H$ is the ocean depth, the ocean surface is at $z=0$, and $U$ is the depth-averaged component of the flow velocity in the direction from the point $A$ to point $B$. Note that the travel time nonreciprocity does not depend on the number of surface reflections and is the same for each eigenray. Within this approximation waveforms are the same for sound propagation with and against the flow.

Using the estimated value of $\tau_{12}$ for $\delta t$, we find $U=-0.47 \mathrm{~m} / \mathrm{s}$. The result obtained from our estimate of $\tau_{23}$ is $5 \%$ higher. The minus sign indicates that the flow is from the instrument 2 to instrument 1, i.e., towards the northeast. This estimate is slightly lower than the deployment cruise ADCP-based estimate, $0.7 \mathrm{~m} / \mathrm{s}$, of the average current in the upper $70 \mathrm{~m}$. We consider this agreement to be good, given that currents in the lower $30 \mathrm{~m}$ are expected to be lower than currents in the upper $70 \mathrm{~m}$. A northeastward depth-averaged current of $\sim 0.5 \mathrm{~m} / \mathrm{s}$ near the $100 \mathrm{~m}$ isobath can be attributed to a meander $[25,26]$ of the Florida current (L. K. Shay, personal communication).

The dominant sources of errors in the $U$ measurement are errors associated with estimation of $\tau_{12}$. These were estimated, as in active tomography (see, e.g., p. 197 in [4]), from the acoustic bandwidth and SNR in the noise cross-correlation function $C_{12}$ (Figure 5a), and were found to be $\sim 0.1 \mathrm{~ms}$. As mentioned above, the accuracy of the clock drift removal assuming linear drift over 1 week is also within $0.1 \mathrm{~ms}$. The expected time shift measurement error is thus approximately $\left(0.1^{2}+0.1^{2}\right)^{1 / 2} \mathrm{~ms}$, corresponding to a current estimation error of $\sim 7 \%$.

\section{Conclusion}

Using simple, single-hydrophone recording systems, we have demonstrated experimentally the feasibility of making passive measurements of acoustic nonreciprocity in the ocean at scales large compared to the ocean depth. Interferometry of low-frequency ambient and shipping noise is shown to provide sub-millisecond accuracy in 
measurements of the acoustic travel time nonreciprocity and to give robust estimates of the depth-averaged flow velocity, despite very limited a priori information about the noise sources and sound propagation conditions. Further research is necessary to improve accuracy and extend the measurements to longer ranges. Anticipated future development of inexpensive low-power atomic clocks should lead to improved accuracy while simplifying the analysis. It is expected that wider frequency bands of ambient noise will prove useful for passive measurements on refracted acoustic paths and in less dynamic environments representative of the deep ocean. Flow velocity measurements complement previously demonstrated [14] passive measurements of the sound speed profile (and, therefore, water temperature) and open the possibility of using acoustic noise interferometry to measure heat fluxes in the ocean.

\section{Competing interests}

The authors declare that they have no competing interests.

\section{Authors' contributions}

OG conceived of the study, participated in data interpretation, and drafted the manuscript. MB designed and carried out the experiments, participated in data processing and analysis, and helped to draft the manuscript. NZ carried out data processing and analysis, participated in data interpretation, and helped to draft the manuscript. LZ carried out data processing and participated in data analysis. NW designed and carried out the experiments. All authors read and approved the final manuscript.

\section{Acknowledgments}

Data processing was performed on the Janus supercomputer, which is supported by the National Science Foundation (award number CNS-0821794) and the University of Colorado Boulder. We thank Jules Hummon of the University of Hawaii Data Acquisition System ADCP group for assistance with processing the ADCP data and preparation of Figure $1 \mathrm{~b}$. Constructive comments by two anonymous referees helped to improve presentation. This work was supported, in part, by the National Science Foundation, grants OCE1129860 and OCE1129524, NAVAIR, award N68335-12-C-0104, and the Office of Naval Research, grant N00014-12-10182.

\section{Author details}

${ }^{1}$ Cooperative Institute for Research in Environmental Sciences, University of Colorado and NOAA Earth System Research Laboratory, Physical Sciences Division, Boulder, Colorado 80305-3328, USA. ${ }^{2}$ Rosenstiel School of Marine and Atmospheric Science, University of Miami, Miami, FL 33149, USA. ${ }^{3}$ Cooperative Institute for Research in Environmental Sciences and Department of Electrical, Computer, and Energy Engineering, University of Colorado, Boulder, Colorado 80309, USA.

Received: 12 September 2014 Accepted: 25 November 2014 Published online: 12 December 2014

\section{References}

1. Howe BM, Worcester PF, Spindel RC (1987) Ocean acoustic tomography: Mesoscale velocity. J Geophys Res 92:3785-3805

2. Ko DS, DeFerrari HA, Malanotte-Rizzoli P (1989) Acoustic tomography in the Florida Strait: Temperature, current, and vorticity measurements. J Geophys Res 94:6197-6211

3. Dushaw BD, Howe BM, Cornuelle BD, Worcester PF, Luther DS (1995) Barotropic and baroclinic tides in the central North Pacific Ocean determined from long-range reciprocal acoustic transmissions. J Phys Oceanogr 25:631-647

4. Munk W, Worcester P, Wunsch C (1995) Ocean acoustic tomography. Cambridge University Press, Cambridge, pp 115-135
5. Godin OA, Mikhin DY, Palmer DR (2000) Monitoring ocean currents in the coastal zone. Izv Atmos Ocean Phys 36:131-142

6. Goncharov W, Ivanov VN, Kochetov OY, Kuryanov BF, Serebryanyi AN (2012) Acoustic tomography at shelf of the Black Sea. Acoust Phys 58:562-570

7. Huang C-F, Yang TC, Liu J-Y, Schindall J (2013) Acoustic mapping of ocean currents using networked distributed sensors. J Acoust Soc Am 134:2090-2105

8. Taniguchi N, Kaneko A, Yuan Y, Gohda N, Chen H, Liao G, Yang C, Minamidate M, Adityawarman Y, Zhu X, Lin J (2010) Long-term acoustic tomography measurement of ocean currents at the northern part of the Luzon Strait. Geophys Res Lett 37, L07601. doi:10.1029/2009GL042327

9. Taniguchi N, Huang CF, Kaneko A, Liu CT, Howe BM, Wang YH, Yang Y, Lin J, Zhu X-H, Gohda N (2013) Measuring the Kuroshio Current with ocean acoustic tomography. J Acoust Soc Am 134:3272-3281

10. Zhu XH, Kaneko A, Wu Q, Zhang C, Taniguchi N, Gohda N (2013) Mapping tidal current structures in Zhitouyang Bay, China, using coastal acoustic tomography. IEEE J Ocean Eng 38:285-296

11. Godin OA, Mikhin DY, Mokhov AV (1996) Acoustic tomography of oceanic currents by matching non-reciprocity. Akust Zhurn 42:501-509

12. Larose E, Margerin L, Derode A, van Tiggelen B, Campillo M, Shapiro N, Paul A, Stehly L, Tanter M (2006) Correlation of random wavefields: An interdisciplinary review. Geophysics 71:SI11-SI21

13. Roux P, Kuperman WA, the NPAL Group (2004) Extracting coherent wave fronts from acoustic ambient noise in the ocean. J Acoust Soc Am 116:1995-2003

14. Godin OA, Zabotin NA, Goncharov W (2010) Ocean tomography with acoustic daylight. Geophys Res Lett 37, L13605. doi:10.1029/2010GL043623

15. Lani SW, Sabra KG, Hodgkiss WS, Kuperman WA, Roux P (2013) Coherent processing of shipping noise for ocean monitoring. J Acoust Soc Am 133:EL108-EL113

16. Yardim C, Gerstoft P, Hodgkiss WS, Traer J (2014) Compressive geoacoustic inversion using ambient noise. J Acoust Soc Am 135:1245-1255

17. Brown MG, Godin OA, Williams NJ, Zabotin NA, Zabotina L, Banker GJ (2014) Acoustic Green's function extraction from ambient noise in a coastal ocean environment. Geophys Res Lett 41:5555-5562. doi:10.1002/2014GL060926

18. Godin OA (2006) Recovering the acoustic Green's function from ambient noise cross-correlation in an inhomogeneous moving medium. Phys Rev Lett 97:054301. doi:10.1121/1.2167148

19. Godin OA (2010) Cross-correlation function of acoustic fields generated by random high-frequency sources. J Acoust Soc Am 128:600-610

20. Godin OA, Irisov VG, Charnotskii MI (2014) Passive acoustic measurements of wind velocity and sound speed in air. J Acoust Soc Am 135:EL68-EL74

21. Godin OA, Zabotin NA, Sheehan AF, Collins JA (2014) Interferometry of infragravity waves off New Zealand. J Geophys Res Oceans 118:1103-1122. doi:10.1002/2013JC009395

22. Stehly L, Campillo M, Shapiro NM (2007) Travel time measurements from noise correlation: Stability and detection of instrumental time-shifts. Geophys J Int 171:223-230

23. Sens-Schönfelder C (2008) Synchronizing seismic networks with ambient noise. Geophys J Int 174:966-970

24. Gouédard P, Seher T, McGuire JJ, Collins JA, van der Hilst RD (2014) Correction of ocean-bottom seismometer instrumental clock errors using ambient seismic noise. Bull Seism Soc Am 104:1276-1288

25. Shay LK, Lee TN, Williams EJ, Graber HC, Rooth CG (1998) Effects of low-frequency current variability on near-inertial submesoscale vortices. J Geophys Res 103:18691-18714

26. Parks AB, Shay LK, Johns WE, Martinez-Pedraja J, Gurgel K-W (2009) HF radar observations of small-scale surface current variability in the Straits of Florida. J Geophys Res 114:C08002. doi:10.1029/2008JC005025

doi:10.1186/s40562-014-0016-6

Cite this article as: Godin et al:: Passive acoustic measurement of flow velocity in the Straits of Florida. Geoscience Letters 2014 1:16. 